\title{
Evidência Tupí-Guaraní para *pw $\rightarrow$ kw
}

\author{
Aryon Dall'Igna Rodrigues
}

1. O Tupinambá ou Tupí Antigo $(\mathrm{T})^{1}$ tem $k w$ e pw como sequências bem estabelecidas de segmentos assilábicos (e não como fonemas unitários labializados: eles ocorrem paralelamente a outras sequências, não só $m w$ e $\eta w$, mas também $r w, n w, s w, ? w e$ py, $k y, m y, \eta y, r y, n y, s y, ? y)$. pwe $k w$ do T correspondem ambos a $k w$ do Guaraní Antigo $(\mathrm{GA})^{2}$ :
(1a) T apwã
(1b) GA akwã
'lábio superior'
(2a) T opwerá $\beta$
GA akwerá
'sarou'
(3a) T syepwár
GA cyekwá
'amarrou-me'
(4a) T syekwár
GA cyekwá
'tenho um buraco'
(5a) T kwesé
$(5 b)$
GA kwehé
'ontem'

2. A maioria das demais línguas da família Tupí-Guaraní apresenta situação análoga à do GA, como, por exemplo, o Guarayo da Bolívia oriental

1. O Tupinambá (Tupí Antigo) foi falado ao longo da costa brasileira nos séculos 16 e 17. As principais fontes para seu conhecimento são Anchieta 1595, Anônimo 1952/1953 (manuscrito de 1611), Araújo 1618 e Figueira 1621. Aqui foram utilizadas as análises fonológicas de Rodrigues 1958 e ms. (1981).

2. O Guaraní Antigo foi falado nos séculos 16, 17 e 18 no sudoeste do Brasil, no Paraguai e no nordeste da Argentina. As principais fontes para seu conhecimento são Aragona 1981 (manuscrito de cerca de 1625), Ruíz de Montoya (1639, 1640a e 1640b) e Restivo (1724). Aqui foi utilizada a análise fonológica de Grannier-Rodrigues 1974. Em GA $k w$ pode ser interpretado como um fonema unitário $k w$ (como foi analisado por Grannier-Rodrigues 1974 e como tem sido analisado em alguns dialetos modernos do Guaraní), visto que nesta língua só as velares podem ser seguidas por $w, r w, n w, s w$, etc. do T, correspondente no GA $a, r, n, c$, etc. 
(Hoeller 1932), o Parintintín do rio Madeira, Amazonas (Betts 1982) e o Tembé do rio Gurupi, Pará e Maranhão (Boudin 1966):

$\begin{array}{llll}\text { Guarayo } & \text { Parintintín } & \text { Tembé } & \\ \text { (6a) okwéra } & \text { (6b) okweráv } & \text { (6c) okweráw } & \text { "sarou" } \\ \text { (7a) čékwá } & \text { (7b) jikwár } & \text { (7c) hekwár } & \text { "amarrou-me" } \\ \text { (8a) čékwár } & \text { (8b) jikwár } & \text { (8c) hekwár } & \text { "tenho um buraco" }\end{array}$

Dada a ampla distribuição geográfica dessas línguas, pode parecer mal a provável a hipótese de que a língua ancestral de toda a família, o Proto-Tupí-Guaraní (PTG) tivesse * $k w$ (ou $k^{w}$ ) e que daí se tivesse derivado o $p w$ do T.

Na discussão que se seque vamos restringir-nos aos dados do T e do GA, já que este último representa a situação de língua como o Guarayo, o Parintintín e o Tembé a vantagem de ser contemporânea do Tupinambá e de prestar-se melhor, por isso, a uma comparação o isenta das complicações que pode apresentar, para a reconstrução, a comparação de línguas de épocas diferentes.

3. Se postularmos para o PTG a sequência * $k w$ (ou o segmento * $k^{w}$ ) como fonte do $\mathrm{T} p w=\mathrm{GA} k w$, teremos para o $\mathrm{T}$ a mudança ${ }^{*} k w \rightarrow p w$, a qual é bastante familiar em diversos domínios linguísticos (Proto-indo-europeu * $k^{w}$ $\rightarrow$ Grego $p$ como er ${ }^{*} l e i k^{w} o: \rightarrow \lambda \varepsilon i ́ \tau \omega$ "deixo" ; latim $k^{w} \rightarrow$ Rumeno $p$ como em aqua $\rightarrow$ apă "água"; etc.). Mas derivar o T $p w$ de um PTG $k w$ põe um problema difícil para a fonologia diacrônica do $\mathrm{T}$, pois obriga a admitir uma cisão arbitrária entre T $p w$ e $k w$ :

(9) PTG *ice kwár T syekwár (GA cyekwá) "tenho um buraco"

(10) PTG*ice kwár T syepwár (GA cyekwá) "amarrou-me"

Se, ao contrário, postularmos $\mathrm{PTG}^{*} p w\left(\mathrm{ou}{ }^{*} p^{w}\right)$ como fonte do T $p w$ e PTG ${ }^{*} k w$ (ou ${ }^{*} k w$ ) como origem do T $k w$, não teremos nenhum problema, pois em vez de uma cisão arbitrária no T, deveremos admitir na história do GA uma fusão absoluta de * $p w$ e * $k w$, o que representa um caso trivial em fonologia diacrônica:

(11) PTG*ice kwár $\rightarrow$ T syekwár, GA cyekwá "tenho um buraco"

(12) PTC *ice pwár $\rightarrow$ T syepwár, GA cyekwá "amarrou-me"

A mudança $p w \rightarrow k w$, embora menos familiar na linguística histórica, não é entretanto incomum. Um caso análogo no essencial - que a substituição de uma articulação labial por uma articulação velar diante de $w$-ocorre, por exemplo, em dialetos hispano-americanos da Argentina e do Paraguai, em que 
$b w \rightarrow g w$ como em buey $\rightarrow$ guey [gwéy] "boi" e $f w \rightarrow x w$ como em fuerte $\rightarrow$ juerte [xwérte] 'forte'.

4. Não só o T $p w$ corresponde ao GA $k w$, mas a mesma relação existe também para as sequências nasais paralelas, T $m w=$ GA $\eta w$ :
(13a) T memwã
(13b) GA meywã
"brincadeira, gracejo"

Portanto, devemos postular também PTG ${ }^{*} m w \rightarrow$ GA $\eta w$ paralelamente a ${ }^{*} p w \rightarrow k w$.

Os $m w$ 's do T que correspondem a $\eta w$ 's do GA são ou lexicais (tautomorfêmicos) como em (13) ou resultantes da junção de $m$ em fim de morfema e $w$ inicial de morfema, portanto heteromorfêmicos, como em (15):

$$
\begin{aligned}
& \text { (14a) T } a \beta a ́+\text { rám }+a \\
& \text { (14b) GA } a \beta a ́+\text { rám }+a \\
& \text { (15a) T aßá + rám + wér }+a \\
& \text { (15b) GA } a \beta a ́+\text { rán+wér }+a
\end{aligned}
$$

"quem vai tornar-se homem"

"quem devia tornar-se homem (mas não se tornou)"

Como se vê nos exemplos (14) e (15), o GA tem uma regra sincrônica que converte $m$ final de morfema em $\eta$ diante de $w$ inicial de morfema seguinte, ao passo que o T mantém inalterado se no mesmo contexto. Essa regra sincrônica do GA $(m+w \rightarrow \eta w)$ é quase idêntica à regra diacrônica ${ }^{*} m w \rightarrow \eta w$ e é, evidentemente, uma consequência desta.

5. Além dos dois casos em que o T $m w$ corresponde ao GA $\eta w$, há um terceiro caso em que o T $m w$ corresponde ao GA $m w$ (e não $\eta w$ ). Em T esse caso resulta quando a sequência $p w$ inicial de morfema é precedida por um segmento nasal final de morfema; em GA a situação é a mesma, mas com $k w$ em vez de $p w$ :
(16a) T ayopwár
(16b) GA ayokwá "eu o amarrei"
(17a) T ayekulápwár
(17b) GA ayeku?ákwá
"amarrei minha cintura"
(18a) T ayepwãmwár
(18b) GA ayekwãmwá
"amarrei meu dedo"
(19a) T ayeakãmwár
(19b) GA ayeakãmwá
"amarrei minha cabeça"
$(\rightarrow i a+y e+a k a \eta+p w a ́ r)$
$(\rightarrow a+y e+a k a ́ \eta+k w a ́ r)$

Nestes exemplos o GA mostra uma alternância morfofonêmica $k w \sim m w$ (no morfema $k w a ́$ mwá "amarrar"), a qual implica uma regra sincrônica determinando que, diante de $w$, a oclusiva $k$ inicial de morfema e substituída pela nasal labial quando precedida por um segmento nasal na mesma palavra, 
mas permanece inalterada em outros contextos. Esta regra, entretanto, não se aplica a todos os casos de $k w$ precedido por segmento nasal, uma vez que há casos em que o GA $k w$ mude em $\eta w$ :

(20a) T apisá + kwár + a (20b) GA apicá + kwá "buraco do ouvido"

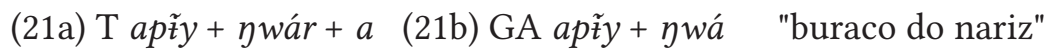

Como se vê em (20) e (21), esses são os casos em que o GA $k w$ corresponde ao T $k w$, e não a $p w$.

Enquanto que a produção de $m w$ em T nos exemplos (18a) e (19a) é bem motivada e tem um paralelo na mudança $k w \rightarrow \eta w$ no exemplo (21a), a mudança do GA $k w \rightarrow m w$ é difícil de explicar sincronicamente; diacronicamente, a hipótese de que PTG ${ }^{*} p w \rightarrow{ }^{*}$ GA $k w$ e PTG ${ }^{*} m w \rightarrow$ GA $\eta w$ a esclarece cabalmente, o que não se dá com a hipótese inversa de que $\mathrm{PTG}^{*} k w \rightarrow \mathrm{T} p w$ e PTG ${ }^{*} \eta w \rightarrow \mathrm{T} m w$.

6. Tanto em T como em GA os nomes possuídos tem uma forma absoluta (ou humana) que, para os temas iniciados por $p$, é marcada pelo prefixo $m$ - e para os temas começados por outras consoantes não é marcado (ou é marcado por $\varnothing$ ) (temas iniciados por vogal tomam $t$ - ou $\varnothing$, ou perdem sua vogal inicial):
(22a) T kunumĩ pó
(22b) GA kunumĩ pó "a mão do menino"
(23a) T $(m+$ pó $\rightarrow)$ mó
(23b) GA $(m+$ pó $\rightarrow)$ mó "mão humana"
(24a) T kunumĩku?á
(24b) GA kunumĩku?á
"cintura do menino"
(25a) T kưá
(25b) GA ku?á
"cintura humana"

A mudança fonológica $m p \rightarrow m$ em (23) é regular em ambas as línguas:

(26a) T kolém + pitáy $+a \rightarrow$ kolémitána "aurora (manhã-vermelho-nominativo)"

(26b) GA ko?ém + pitán $\rightarrow$ ko?émitã $\quad$ "aurora (manhã-vermelho)"

A palavra T para "dedo", $p w a \tilde{a}$, comporta-se como os demais nomes possuídos que começam por $p$ :

(27) kunumĩ kwã

"o dedo do menino"

(28) $m w \tilde{a}$

"dedo humano"

A palavra correspondente em GA parece irregular em comparação com os exemplos (22b) - (23b), mas é tão regular quanto os exemplos (16b) - (19b): 
(29) GA kunumĩ kwá

(30) GA $m w \tilde{a}$ "dedo do menino"

"dedo humano"

A irregularidade paradigmática sincrônica do GA $k w a \tilde{a}$ "dedo", que aparentemente mistura os paradigmas regulares de ku?á "cintura" e pó "mão", tem claramente origem e explicação na mudança diacrônica PTG * $p w \rightarrow$ GA $k w$. Enquanto o PTG pwã converteu-se em GA $k w a ́$, o PTG * $m+$ pwá converteu-se em GA mwá da mesma forma como o PTG *-akán + pwár resultou no GA akãmwá (19b).

7. Todos os fatos discutidos acima falam em favor de uma regra diacrônica ${ }^{*} p w / m w \rightarrow k w / \eta w$ na história do GA e não em favor de uma regra ${ }^{*} k w / \eta w \rightarrow$ $p w / m w$ na história do T. Um resumo informal das regras diacrônicas envolvidas na derivação do GA $k w$ e $\eta w$ poderia ser apresentado assim:

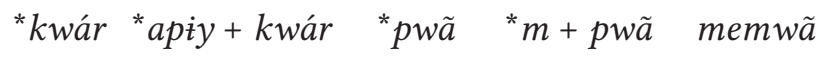

(31) Epêntese de nasal apz̃yjkwár

(32) Velarização de labiais entre $\mathrm{V}$ ou $k w \tilde{a}$ mẽywã \# e w

(33) Redução de mp/yk a $\mathrm{m} / \mathrm{y}^{3}$

apiyywár $m w \tilde{a}$

Outras regras

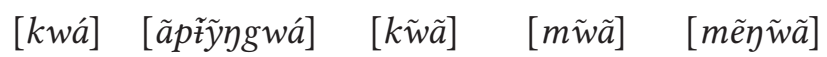

\section{Bibliografia}

Anchieta, Joseph de. 1595. Arte de grammatica da Língua mais usada no Costa do Brasil. Coimbra.

Anônimo. 1952/1953. Vocabulário na Língua Brasilica. $2^{\mathrm{a}}$ edição revista e confrontada com o ms. fg. 3144 da Bibl. Nacional de Lisboa por Carlos Drummond. (Univer-

3 A aplicação da regra (33) parece inconsistente com o produto fonético final na derivação de [ãpz̃̃y gwá]. Prefiro representar assim, uma vez que há uma regra mais superficial que cinde todo segmento nasal em duas manifestações fonéticas unissegmentais independentemente de sue fonte histórica (seja esta * $m$ ou * $m p$ ), a saber, uma nasal plena diante de vogais nasais e uma "pré-nasa1izada" diante de vogais orais (p. ex., " mo+yán $\rightarrow \mathrm{CA}$

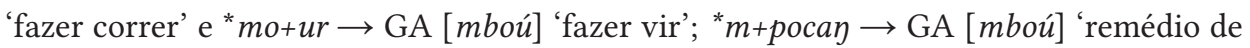
gente', * $m+$ poti?á $\rightarrow$ GA [mboti?á] 'peito humano'. 
sidade de São Pau1o, Faculdade de Filosofia, Ciências e Letras, Boletim n ${ }^{\circ}$ 137, Etnografia e Tupi-Guarani no 23). São Paulo. 2 vols.

Araujo, Antonio d'. 1618. Catecismo na Lingoa Brasilica... Agora novamente concertado, ordenado, e acrescentado pello Padre... Lisboa.

Fiqueira, Luis. 1621. Arte da língua brasílica. (2a. edição: Arte de grammatica da língua brasilica, Lisboa, 1687.)

Grannier-Rodrigues, Daniele Marcelle. 1974. Fonologia do Guaraní Antigo. Dissertação de Mestrado, Universidade Estadual de Campinas.

Rodrigues, Aryon Dall'lgna. 1958. Phonologie der Tupinambá-Sprache. Tese de doutorado, Universität Hamburg.

Ruiz (de Montoya), Antonio. 1639. Tesoro de la lengua guarani. Madrid.

Ruiz (de Montoya), Antonio. 1640a. Arte, y bocabulario de la lengua guarani. Madrid.

Ruiz (de Montoya), Antonio. 1640b. Catecismo de la Lengua Guarani. Madrid. 